\title{
AN INNOVATION DIFFUSION MODEL FOR THE SURVIVAL OF A PRODUCT IN A COMPETITIVE MARKET: BASIC INFLUENCE NUMBERS
}

\author{
Joydip Dhar ${ }^{1}$, Mani Tyagi ${ }^{2}$, P. Sinha ${ }^{3}$ \\ ${ }^{1}$ ABV-Indian Institute of Information Technology and Management \\ Gwalior (M.P.), INDIA \\ ${ }^{2,3}$ Department of Mathematics \\ Govt. Model Science College \\ Gwalior, 474015 (M.P.), INDIA
}

\begin{abstract}
In this paper, we propose a compartmental model to study the influence of word of-mouth to the spreading of information of new product with the market dynamics with two competitive products in a given closed region. The model was analyzed qualitatively to determine the possible steady states and it's local stability under the influence of personal interaction. Here, we calculated the basic influence numbers(BINs) for both the products and combined BIN of the proposed system. It is observed that adopter free equilibrium is locally stable only when the BINs are less than one for both the product and interior equilibrium is always unstable, hence in long run the co-existence of both the product is the market is impossible. Further, it is also shown that value of BINs will determine the survival of a particular product. Finally, numerical justification of the analytical results are performed with different set of values and presented graphically in the numerical section.
\end{abstract}

\section{AMS Subject Classification: 91FXX, 34CXX, 34DXX}

Key Words: innovation diffusion, compartment model, basic influence number, local stability

Received: $\quad$ March 13, 2013

(C) 2013 Academic Publications, Ltd.

${ }^{\S}$ Correspondence author url: www.acadpubl.eu 


\section{Introduction}

The new product diffusion model is mainly applied to conducting forecasting for product life cycles and trend of product purchases. After analyzing the model, manufacturers can be informed of which period of the life cycle the product will enter into the market, as well as how the customer will make decisions in certain periods. Since Bass[1] carried out assessments of new products in terms of the quantitative model, had been utilized to investigate diffusion patterns and demand forecasting. Afterwards, many studies, based on the Bass Model, endeavored to expand, incorporate, modify and introduce new methods for the purpose of reflecting the complexity of the market. Jain and Rao [2] suggested that the price of the product had an effect on the whole market potential, while Jones and Ritz [3] claimed that an increase in the number of the retailers would affect the whole market. Lilien et al.[4] verified the medicines diffusion situation with the product repeat-purchase model. Norton and Bass [5] declared the multi-generation substitute diffusion model and applied it to obtain high-technology products substitution and diffusion for each successive generation. However, most previous diffusion models extended from Bass [1] assumed a monopolistic market, which in reality process different characteristic of interactive competitive markets. The LotkaVolterra equation, which was developed to model the interaction between two competing species based on the logistic curve, has been adopted to the situation of competitive markets. Modis [6] analyzed the behaviour of common stock-bond interactions by the LotkaVolterra model, as if they were species competing for investors resources. Furthermore, Modis [7] focused on the topic of genetic re-engineering of corporations for discussion, described the competitive dynamics in a market niche occupied by two competitors, and classified the types of competitive roles according to the models parameter. The global stability of equilibria of an innovation diffusion model for two and three competitive products respectively studied [15, 16]. Lee et al.[8] analyzed the dynamic competitive relationship on the Korean stock market, and verified that the competitive roles would change as time passed. On the other hand, Maurer and Huberman [10] adopted the LotakVolterra model to explore the effects of competition among web sites. Furthermore, Kim et al. [11] explored the Korean mobile phone market and found a commensalism relationship, while Lope and Sanjuan [12] analyzed the competition dynamics of web sites on the internet, which are useful for defining strategies on the internet market.

In this paper, we propose a deterministic mathematical model to study the dynamical behavior of a system in which two competitive products in a 


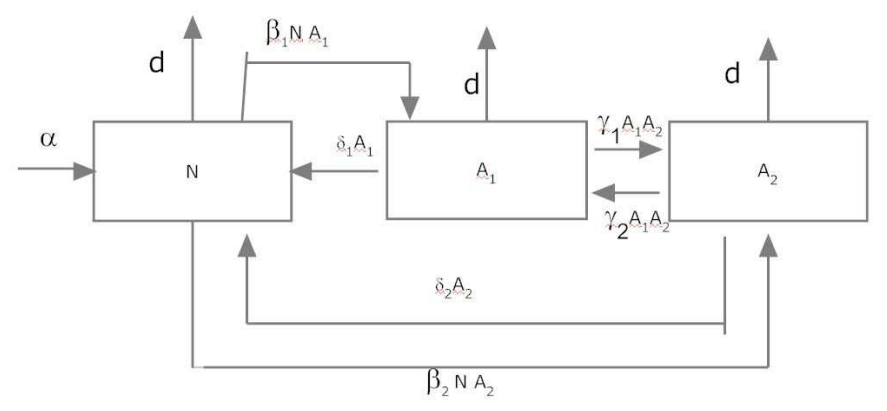

Figure 1: Schematic model for two competitive product

potential market. The organization of this paper is: in Section 2, a deterministic mathematical model formulation is developed, with two competing product under the internal influence to the spreading of the adopter. In Section 3, calculation of basic influence numbers and study of local asymptotical stability of the all equilibria are presented. Finally numerical validation and a brief conclusions are presented in Section 4 and 5 respectively.

\section{Mathematical Model}

Here, we assumed that the any given market system population is divided into three categories, namely, the non-users of the population $N(t)$, adopter population of first product $A_{1}(t)$ and adopter population of second product $A_{2}(t)$ at any time $t$. Further, it is assumed that only mouth-mouth spreading of the particular new product and each non-user will adopts only one product at a time. Let $\alpha$ is the new recruitment rate of population which will join the system as non-adopter and without loss of generality, assume that $d$ is the constant nature death rates of all categories of population. Let $\beta_{1}, \beta_{2}$ be the valid contact rate of non-users with users of first and second products respectively. Assume that $\delta_{i}, i=1,2$ are the frustration rates of $i$ th adopter population, which will join non-users class. Again $\gamma_{1}$ and $\gamma_{2}$ are the competition rates between the adoptions. The schematic flow the propose model is shown in figure 1. Taking the market dynamics as continuous, our proposed model is govern by the following set of differential equations:

$$
\frac{d N}{d t}=\alpha-\beta_{1} N A_{1}-\beta_{2} N A_{2}+\delta_{1} A_{1}+\delta_{2} A_{2}-d N .
$$




$$
\begin{aligned}
& \frac{d A_{1}}{d t}=\beta_{1} N A_{1}-\delta_{1} A_{1}-\gamma_{1} A_{1} A_{2}-d A_{1} . \\
& \frac{d A_{2}}{d t}=\beta_{2} N A_{2}-\delta_{2} A_{2}-\gamma_{2} A_{1} A_{2}-d A_{2} .
\end{aligned}
$$

with initial populations are $N(0)=N_{0}, A_{1}(0)=A_{10}$ and $A_{2}(0)=A_{20}$. It follows from system (1)-(3) that

$$
\frac{d}{d t}\left(N+A_{1}+A_{2}\right) \leq \alpha-d\left(N+A_{1}+A_{2}\right)
$$

and $\limsup _{t \rightarrow \infty}\left(N+A_{1}+A_{2}\right) \leq \frac{\alpha}{d}$. Hence the system is bounded above. Thus the feasible region for system is $\Omega=\left\{\left(N, A_{1}, A_{2}\right): 0 \leq N+A_{1}+A_{2} \leq \frac{\alpha}{d}, N \geq\right.$ $\left.0, A_{1} \geq 0, A_{2} \geq 0\right\}$. Let Int $\Omega$ denote the interior of $\Omega$. It is easy to verify that the region $\Omega$ is a positively invariant(i.e., the solution with initial conditions in $\Omega$ remain in $\Omega$ ) with respect to system (1)-(3).

In the next section, firstly, we will calculate the basic influence number(BIN) of system (1)-(3). Secondly, we will obtain the stability conditions around all feasible equilibrium in term of BIN.

\section{System Dynamics}

Here, we will be defining one useful threshold parameters which characterize mathematical problems concerning new product adoptions is the basic influence number or basic influence rate or basic influence ratio. The basic influence number $R_{A}$, is defined as the expected number of secondary adoption caused by an adopter individual upon entering a totally non-adopter population, which is similar to the basic reproduction number in disease spreading models [14]. This metric is useful because it helps determine whether or not the products will spread through a population. As the system (1)-(3) always has a adopterfree equilibrium, i.e., $E_{1}\left(\frac{\alpha}{d}, 0,0\right)$. Using the same notations in $[13,14]$, we have two vectors $F$ and $V$ to represent the new adopters from the direct contact adopter population with non-adopter population and remaining transfer terms, respectively from system equations of adopter compartments [i.e., (2)-(3)], we have

$$
\mathcal{F}=\left(\begin{array}{c}
\beta_{1} A_{1} N \\
\beta_{2} A_{2} N
\end{array}\right), \quad \mathcal{V}=\left(\begin{array}{c}
\delta_{1} A_{1}+\gamma_{1} A_{1} A_{2}+d A_{1} \\
\delta_{2} A_{2}+\gamma_{2} A_{1} A_{2}+d A_{2}
\end{array}\right)
$$

Hence a straightforward calculation of jacobian matrices around the adopterfree equilibrium $E_{1}$ gives

$$
F=J(\mathcal{F})_{E_{1}}=\left(\begin{array}{cc}
\frac{\beta_{1} \alpha}{d} & 0 \\
0 & \frac{\beta_{2} \alpha}{d}
\end{array}\right), \quad V=J(\mathcal{V})_{E_{1}}=\left(\begin{array}{cc}
\delta_{1}+d & 0 \\
0 & \delta_{1}+d
\end{array}\right)
$$


where $F$ is non-negative and $V$ is a non-singular M-matrix, therefore $F V^{-1}$ is non-negative, and

$$
V^{-1}=\left(\begin{array}{cc}
\frac{1}{\delta_{1}+d} & 0 \\
0 & \frac{1}{\delta_{2}+d}
\end{array}\right), \quad F V^{-1}=\left(\begin{array}{cc}
\frac{\beta_{1} \alpha}{d\left(\delta_{1}+d\right)} & 0 \\
0 & \frac{\beta_{2} \alpha}{d\left(\delta_{2}+d\right)}
\end{array}\right)
$$

Now, $F V^{-1}$ is the next generation matrix for model (1)-(3). It then follows that the spectral radius of matrix $F V^{-1}$ is $\rho\left(F V^{-1}\right)$. According to Theorem 2 in [13], the basic influence number of model ((1)-(3) denoted by $\mathcal{R}_{A}$, is given $\rho\left(F V^{-1}\right)$, where $\mathcal{R}_{A}=\operatorname{Max}\left(\mathcal{R}_{A_{1}}, \mathcal{R}_{A_{2}}\right)$. It can be noted that $\mathcal{R}_{A_{1}}$ and $\mathcal{R}_{A_{2}}$ are the associated basic influence number for the adopters $A_{1}$ and $A_{2}$, respectively, and are given by $\mathcal{R}_{A_{1}}=\frac{\alpha \beta_{1}}{d\left(d+\delta_{1}\right)}$ and $\mathcal{R}_{A_{2}}=\frac{\alpha \beta_{2}}{d\left(d+\delta_{2}\right)}$.

The system (1)-(3) having four feasible steady states, namely:

1. $E_{1}\left(\frac{\alpha}{d}, 0,0\right)$ : Both adopter competitors population are zero, and only nonadopter will be survive in the environment.

2. $E_{2}\left(\frac{d+\delta_{1}}{\beta_{1}}, \frac{\left(d+\delta_{1}\right)}{\beta_{1}}\left[\frac{\alpha \beta_{1}}{d\left(d+\delta_{1}\right)}-1\right], 0\right):$ Corresponds to a situation where $N$ and $A_{1}$ will survive in the environment and $A_{2}$ will disappear. $E_{2}$ is exists $\frac{\alpha \beta_{1}}{d\left(d+\delta_{1}\right)}=\mathcal{R}_{A_{1}}>1$.

3. $E_{3}\left(\frac{d+\delta_{2}}{\beta_{2}}, 0, \frac{\left(d+\delta_{2}\right)}{\beta_{2}}\left[\frac{\alpha \beta_{2}}{d\left(d+\delta_{2}\right)}-1\right]\right)$ : Corresponds to a situation where $N$ and $A_{2}$ will survive in the environment and $A_{1}$ will disappear. $E_{3}$ is exists only when $\frac{\alpha \beta_{2}}{d\left(d+\delta_{2}\right)}=\mathcal{R}_{A_{2}}>1$.

4. $E_{4}\left(\hat{N}, \hat{A}_{1}, \hat{A}_{2}\right)$ : The positive interior equilibrium point is obtained, where $\hat{A_{1}}=\frac{-d+\hat{N} \beta_{2}-\delta_{2}}{\gamma_{2}}, \hat{A_{2}}=\frac{-d+\hat{N} \beta_{1}-\delta_{1}}{\gamma_{1}}$ and $\hat{N}$ is given by

$$
B_{1} \hat{N}^{2}+B_{2} \hat{N}+B_{3}=0
$$

where $B_{1}=\frac{\beta_{1} \beta_{2}\left(\gamma_{1}+\gamma_{2}\right)}{\gamma_{1} \gamma_{2}}, B_{2}=-\frac{d\left(\beta_{1} \gamma_{1}+\left(\beta_{2}-\gamma_{1}\right) \gamma_{2}\right)+\left(\gamma_{1}+\gamma_{2}\right)\left(\beta_{2} \delta_{1}+\beta_{1} \delta_{2}\right)}{\gamma_{1} \gamma_{2}}$ and $B_{3}=\frac{\delta_{1}\left(d+\delta_{2}\right)}{\gamma_{2}}\left[1-\mathcal{R}_{A_{2}}\right]+\frac{\delta_{2}\left(d+\delta_{1}\right)}{\gamma_{1}}\left[1-\mathcal{R}_{A_{1}}\right]+\frac{\alpha \beta_{2} \delta_{1}}{d \gamma_{2}}+\frac{\alpha \beta_{1} \delta_{2}}{d \gamma_{1}}$.

Let $\mathcal{R}_{A}=\max \left[\mathcal{R}_{A_{1}}, \mathcal{R}_{A_{2}}\right]$ and since $B_{1}>0$, we can state the following lemmas:

Lemma 1. When $\mathcal{R}_{A}<1$, then $B_{3}>0$ and hence the model(1)-(3) has

(i) no interior equilibrium if $B_{2}>0$

(ii) two interior equilibrium if $B_{2}<0$ and $B_{2}^{2}-4 B_{1} B_{2}>0$.

Lemma 2. When $\mathcal{R}_{A}>1$, then the model(1)-(3) has

(i) unique interior equilibrium if $B_{3}<0$.

(ii)two interior equilibrium if $B_{3}>0, B_{2}<0$ and $B_{2}^{2}-4 B_{1} B_{2}>0$.

(iii)no interior equilibrium if $B_{3}>0, B_{2}>0$. 
Now, we will study the local stability around $E_{i}$ and the general variational matrix [9], corresponding to the system is given by

$$
J=\left(\begin{array}{ccc}
-d-A_{1} \beta_{1}-A_{2} \beta_{2} & -N \beta_{1}+\delta_{1} & -N \beta 2+\delta 2 \\
A_{1} \beta_{1} & -d+N \beta_{1}-A_{2} \gamma_{1}-\delta_{1} & -A_{1} \gamma_{1} \\
A_{2} \beta_{2} & -A_{2} \gamma_{2} & -d+N \beta_{2}-A_{1} \gamma_{2}-\delta_{2}
\end{array}\right) .
$$

The characteristic equation about the equilibrium $E_{1}\left(\frac{\alpha}{d}, 0,0\right)$ is given by

$$
(d+\lambda)\left[\left(\left(d+\delta_{1}\right)\left(\mathcal{R}_{A_{1}}-1\right)-\lambda\right)\right]\left[\left(\left(d+\delta_{2}\right)\left(\mathcal{R}_{A_{2}}-1\right)-\lambda\right)\right]=0 .
$$

The eigenvalues of the characteristic equation of $E_{1}$ are $\lambda_{1}=-d, \lambda_{2}=(d+$ $\left.\delta_{1}\right)\left(\mathcal{R}_{A_{1}}-1\right)$ and $\lambda_{3}=\left(d+\delta_{2}\right)\left(\mathcal{R}_{A_{2}}-1\right)$. Thus all the eigenvalue of equation (7) having negative real parts when both $\mathcal{R}_{A_{1}}<1$ and $\mathcal{R}_{A_{2}}<1$. Therefore the equilibrium point $E_{1}$ is always locally asymptotically stable for $\mathcal{R}_{A_{1}}<1$ and $\mathcal{R}_{A_{2}}<1$.

Again the characteristic equation about the equilibrium $E_{2}$ has eigenvalues $\lambda_{1}=-d, \lambda_{2}=-\left(d+\delta_{1}\right)\left(\mathcal{R}_{A_{1}}-1\right), \lambda_{3}=\left(\left(d+\delta_{2}\right)\left(\mathcal{R}_{A_{2}}-1\right)-\frac{\left(d+\delta_{1}\right)\left(\beta_{2}+\gamma_{2}\right)\left(\mathcal{R}_{A_{1}}-1\right)}{\beta_{1}}\right)$ and all the eigenvalues having negative real parts if $\mathcal{R}_{A_{2}}<1+K_{1}\left(\mathcal{R}_{A_{1}}-1\right)$, where $K_{1}=\frac{\left(d+\delta_{1}\right)\left(\beta_{2}+\gamma_{2}\right)}{\beta_{1}\left(d+\delta_{2}\right)}$ and $\mathcal{R}_{A_{1}}>1$ holds. Similarly the characteristic equation about the equilibrium $E_{3}$ has eigenvalue $\lambda_{1}=-d, \lambda_{2}=-\left(d+\delta_{2}\right)\left(\mathcal{R}_{A_{2}}-1\right)$, $\lambda_{3}=\left(d+\delta_{1}\right)\left(\mathcal{R}_{A_{1}}-1\right)-\frac{\left(d+\delta_{2}\right)\left(\beta_{1}+\gamma_{1}\right)\left(\mathcal{R}_{A_{2}}-1\right)}{\beta_{2}}$ and has negative real parts when both $\mathcal{R}_{A_{1}}<1+K_{2}\left(\mathcal{R}_{A_{2}}-1\right)$, where $K_{2}=\frac{\left(d+\delta_{2}\right)\left(\beta_{1}+\gamma_{1}\right)}{\beta_{2}\left(d+\delta_{1}\right)}$ and $\mathcal{R}_{A_{2}}>1$ satisfies. Finally, the characteristic equation about the equilibrium $E_{4}\left(\hat{N}, \hat{A_{1}}, \hat{A_{2}}\right)$ is given by

$$
\lambda^{3}+a_{1} \lambda^{2}+a_{2} \lambda+a_{3}=0 .
$$

where $a_{1}=d+\hat{A_{1}} \beta_{1}+\hat{A}_{2} \beta_{2}, a_{2}=\hat{A_{1}} d \beta_{1}+\hat{A}_{2} d \beta_{2}+\hat{A_{1}} \hat{A}_{2} \beta_{1} \gamma_{1}+\hat{A_{1}} \hat{A}_{2}\left(\beta_{2}-\gamma_{1}\right) \gamma_{2}$ and

$a_{3}=-\hat{A_{1}} \hat{A_{2}} \hat{N} \delta_{1} \beta_{2} \gamma_{1}-\hat{A_{1}} \hat{A_{2}} \hat{N} \delta_{1} \beta_{2} \gamma_{2}-\hat{A_{1}} \hat{A}_{2} d \gamma_{1} \gamma_{2}-\hat{A}_{1}^{2} \hat{A}_{2} \beta_{1} \gamma_{1} \gamma_{2}-\hat{A_{1}} \hat{A}_{2}{ }^{2} \beta_{1} \gamma_{1} \gamma_{2}+$ $\hat{A_{1}} \hat{A}_{2} \beta_{2} \gamma_{1} \delta_{1}+\hat{A}_{1} \hat{A}_{2} \beta_{1} \gamma_{2} \delta_{2}$. Here $a_{1}>0$ and using existence conditions $\delta_{1}=$ $-\hat{A}_{2} \gamma_{1}-d+\hat{N} \beta_{1}$ and $\delta_{2}=-\hat{A_{1}} \gamma_{2}-d+\hat{N} \beta_{2}$ we get $a_{3}=-\hat{A_{1}} \hat{A}_{2}\left(d \beta_{2} \gamma_{1}+\right.$ $\left.d\left(\beta_{1}+\gamma_{1}\right) \gamma_{2}+\left(\gamma_{1}+\gamma_{2}\right)\left(\hat{A}_{2} \beta_{2} \gamma_{1}+\hat{A}_{1} \beta_{1} \gamma_{2}\right)\right)<0$. Hence from the Routh-Hurwitz Criterion the system is always unstable around $E_{4}$. Since Interior equilibrium is unstable, so either $E_{1}, E_{2}$ or $E_{3}$ will be stable. We can summarize all findings in the following theorem:

Theorem 1. The dynamic behavior of the system (1)-(3):

1. The adopter free equilibrium $E_{1}$ is stable only when $\mathcal{R}_{A_{1}}<1$ and $\mathcal{R}_{A_{2}}<$ 1. 
2. $E_{2}$ is stable, i.e., only the users of first product will survive, when $\mathcal{R}_{A_{1}}>1$ and $\mathcal{R}_{A_{2}}<1+K_{1}\left(\mathcal{R}_{A_{1}}-1\right)$.

3. $E_{3}$ is stable, i.e., only the users of second product will survive, when $\mathcal{R}_{A_{2}}>1$ and $\mathcal{R}_{A_{1}}<1+K_{2}\left(\mathcal{R}_{A_{2}}-1\right)$.

4. The interior equilibrium $E_{4}$ is always unstable.

Therefore form the theorem 1, we can conclude that in long run at the most only one product will be sustained in the market. Now, we will establish this result through numerical experimentations in the next section.

\section{Numerical Simulations}

In this section, we will verify the analytical findings using numerical simulation with different set of parametric values, which are listed in table 1. Firstly, calculated the values of $\mathcal{R}_{A_{1}}, \mathcal{R}_{A_{2}}, X=1+K_{1}\left(\mathcal{R}_{A_{1}}-1\right), Y=1+K_{2}\left(\mathcal{R}_{A_{2}}-1\right)$ for each set of parametric values and checked the stability conditions and the behavior from theorem 1, results are summarized in table 2 and graphically shown in figures 2-3.

Table 1: Values of different parameters

\begin{tabular}{|c|c|c|c|c|c|c|c|c|}
\hline Set No. & $\alpha$ & $\beta_{1}$ & $\beta_{2}$ & $\gamma_{1}$ & $\gamma_{2}$ & $d$ & $\delta_{1}$ & $\delta_{2}$ \\
\hline Set-1 & 0.3 & 0.15 & 0.25 & 0.10 & 0.15 & 0.2 & 0.1 & 0.2 \\
\hline Set-2 & 0.75 & 0.15 & 0.25 & 0.10 & 0.15 & 0.2 & 0.1 & 0.2 \\
\hline Set-3 & 0.8 & 0.10 & 0.10 & 0.10 & 0.15 & 0.2 & 0.1 & 0.2 \\
\hline Set-4 & 0.8 & 0.30 & 0.25 & 0.20 & 0.25 & 0.2 & 0.3 & 0.2 \\
\hline
\end{tabular}

Table 2: Stable of equilibrium w.r.t. $\mathcal{R}_{A_{1}}, \mathcal{R}_{A_{2}}, X, Y$

\begin{tabular}{|c|c|c|c|c|c|c|}
\hline Parameters & $\mathcal{R}_{A_{1}}$ & $\mathcal{R}_{A_{2}}$ & $\mathrm{X}$ & $\mathrm{Y}$ & Stable equilibrium & Figure \\
\hline Set-1 & 0.50 & 0.9375 & -0.5 & 0.9375 & $E_{1}=(1.5,0,0)$ & $2(\mathrm{a})$ \\
\hline Set-2 & 1.25 & 2.3437 & 1.75 & 2.43 & $E_{3}=(1.6,02.15)$ & $2(\mathrm{~b})$ \\
\hline Set-3 & 1.3 & 1 & 1.6250 & 1 & $E_{2}=(3.0,1.0,0)$ & $3(\mathrm{a})$ \\
\hline Set-4 & 2.4 & 2.5 & 3.9167 & 3.40 & $\begin{array}{c}E_{2}=(1.666,2.33,0) \\
E_{3}=(1.6,0,2.4) \\
\text { w.r.t initial value }\end{array}$ & $\begin{array}{c}3(\mathrm{~b}) \\
3(\mathrm{c})\end{array}$ \\
\hline
\end{tabular}



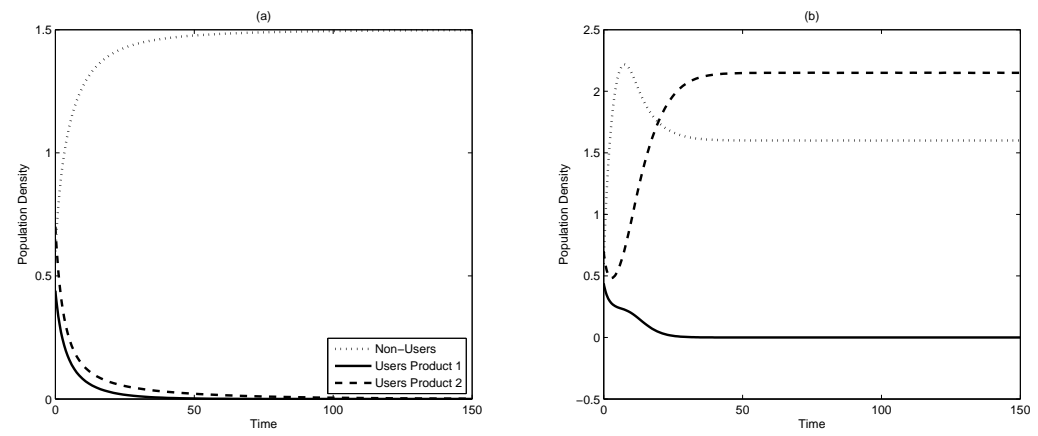

Figure 2: Population Distributions w.r.t time: (a) user-free equilibrium and (b) user of product-1 vanishes

\section{Conclusion}

The new competitive product to sustain itself there must be positive advertisement, personal interaction, immigration and emigration. Keeping in mind the closed market scenario, we discuss a mathematical model with two competing products and non-user population. It is observed that the system having four feasible equilibrium, also examined the local stability of each equilibria. It is observed that the adopter free equilibrium $E_{1}$ is stable if the demand of both the products are vanish from the market. Other two boundary equilibrium $E_{2}$ and $E_{3}$ are also locally asymptotically stable under certain conditions state in theorem 1. Since the interior equilibrium $E_{4}$ is always unstable, hence the co-existence of the users of both products is not possible at the steady state. Therefore either no product or at the most one product will sustain the market in long run.

\section{References}

[1] F.M. Bass, A new product growth model for consumer durable, Manage.Sci., 15, No. 5 (1969), 215-227.

[2] D.C. Jain, R.C. Rao, Effect of price on the demand for durables: Modeling, estimation, and findings, J. Bus. Econ. Stat., 8, No. 2 (1990), 163-170.

[3] J.M. Jones, C.J. Ritz, Incorporating distribution into new product diffusion models, Int. J. Res. Mark., 8, No. 2 (1991), 91-112. 

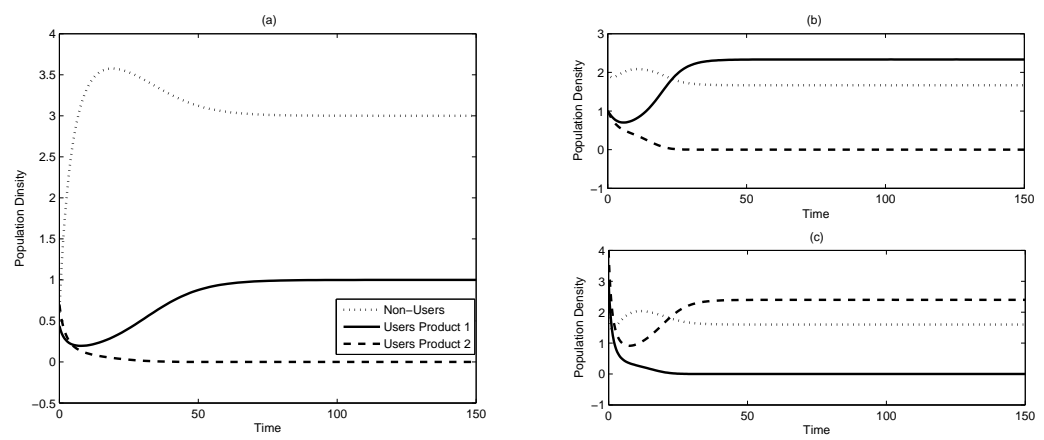

Figure 3: Population Distributions w.r.t time: (a) user of product-2 vanishes, (b) user of product-2 vanishes with initial population densities $(2,1,1)$ and (c) user of product-1 vanishes with initial population densities $(2,3,4)$

[4] G.L. Lilien, A.G. Rao, S. Kalish, Bayesian estimation and control of detailing effort in a repeat purchase diffusion environment, Manage. Sci., 27, No. 5 (1981), 493-506.

[5] J.A. Norton, F.M. Bass, A diffusion theory model of adoption and substitution for successive generations of high-technology products, Manage. Sci., 33, No. 9 (1987), 1069-1086.

[6] T. Modis, Technological forecasting at the stock market, Technol. Forecast. Soc. Change, 62 (1999), 173-202.

[7] T. Modis, Genetic re-engineering of corporations, Technol. Forecast. Soc. Change, 56 (1997), 107-118.

[8] S.J. Lee, D.J. Lee, H.S. Oh, Technological forecasting at the korean stock market: A dynamic competition analysis using Lotka-Volterra model, Technol. Forecast. Soc. Change, 72 (2005), 1044-1057.

[9] J. Dhar, A.K. Sharma, The role of viral infection in phytoplankton dynamics with the inclusion of incubation class, Nonlinear Analysis: Hybrid Systems, 4, No. 1 (2010), 9-15.

[10] S.M. Maurer, B.A. Huberman, Competitive dynamics of web sites, J. Econ. Dyn. Control, 27 (2003), 2195-2206. 
[11] J. Kim, D.J. Lee, J. Ahn, A dynamic competition analysis on the korean mobile phone market using competitive diffusion model, Comput. Ind. Eng., 51 (2006), 174-182.

[12] L. Lopez, M.F.A. Sanjuan, Defining strategies to win in the Internet market, Phys. A, 301 (2001), 512-534.

[13] P.Van den Driwssche, J.Watmough, Reproduction numbers and subthreshold endemic equilibria for compartmental models of disease transmission, Math. Biosci., 180 (2002), 29-48.

[14] G.P. Sahu, J. Dhar, Analysis of an SVEIS epidemic model with partial temporary immunity and saturation incidence rate, Appl. Math. Model., 36, No. 3 (2012), 908-923.

[15] M.C.Kekana, O. D. Makind, A deterministic model for competitive products in a market system, Am. J. Undergrad. Res., 10, No. 2 (2011).

[16] Y. Yu, W. Wang, Y. Zhang, An innovation diffusion model for three competitive products, Computer \& Math with Appli., 46 (2003), 1473-1481. 